\title{
Capacidad entomocida de cepas nativas de Beauveria sp. sobre Schistocerca piceifrons peruviana (Lynch Arribalzaga, 1903)
}

\author{
Entomocidal ability of native strains Beauveria sp. on Schistocerca picei- \\ frons peruviana (Lynch Arribalzaga, 1903) \\ Nicolaza Pariona, Pedro Castellanos y Elizabeth León
}

Facultad de Ciencias Biológicas, Universidad Nacional Mayor de San Marcos, Av. Venezuela cdra $35 \mathrm{~s} / \mathrm{n}$ Ciudad Universitaria. Apartado 110058 Lima 11, Perú.

Email Pedro Castellanos: pcastellanoss@unmsm.edu.pe

Presentado: $\quad 13 / 02 / 2007$ Aceptado: $\quad 20 / 07 / 2007$

\begin{abstract}
Resumen
Cuatro cepas de Beauveria sp. (ABvPr11, ABvPr8, ABvPr3 y ABvSr4), fueron aisladas y caracterizadas de ejemplares de la langosta Schistocerca piceifrons peruviana procedentes de la Provincia de Huamanga del Departamento de Ayacucho. La capacidad entomocida de las 4 cepas fue evaluada en la langosta Schistocerca piceifrons peruviana. Los resultados muestran que todas las cepas, en soluciones de $10^{8}$ conidias $/ \mathrm{mL}$, producen un $100 \%$ de mortalidad. El tiempo que se necesitó para eliminar el $100 \%$ de las langostas, para las cepas ABvPr11, ABvPr8, ABvSr4 y ABvPr3 fue de 12,14 y 16 días respectivamente. Al décimo día se observó diferencias significativas respecto a la mortalidad, entre la cepa ABvPr11(90\%) y la cepas ABvSr4(74\%), ABvPr8(72\%) y ABvPr3(64.6\%). Posteriormente se determinó el tiempo de letalidad al $50 \%\left(\mathrm{TL}_{50}\right)$ de la población de langostas, con un intervalo de 8,38 a 9,16 días y el tiempo de letalidad al $80 \%\left(\mathrm{TL}_{80}\right)$ fue de 9,6 a 11,5 días. La cepa ABvPr11 presentó la mayor actividad entomocida para el control de Schistocerca piceifrons peruviana L.A.
\end{abstract}

Palabras claves: entomopatogeno, control biológico, langosta, Schistocerca, Beuaveria.

\begin{abstract}
Four strains of Beauveria sp. (ABvPr11, ABvPr8, ABvPr3, ABvSr4), that were found in the locust Schistocerca piceifrons peruviana from Huamanga, Ayacucho, Peru, were isolated and morphologically characterized. Entomicidal activities of four strains were evaluated on the locust. Results showed that all the strains producing $100 \%$ mortality when using solutions of 108 conidias $/ \mathrm{mL}$. The time for $100 \%$ mortalities of the strains ABvPr11, ABvPr8, ABvSr4 y ABvPr3 were 12, 14, 14 y 16 days respectively. In the tenth day significant differences were observed between the mortality of the strains ABvPr11 (90\%) and ABvSr4 (74\%), ABvPr8 (72\%), ABvPr3 (64,6\%). Lethality time was determinate for the $50 \%\left(\mathrm{TL}_{50}\right)$ of the locust population: ranging from 8,38 to 9,16 days. Lethality time for the $80 \%\left(\mathrm{TL}_{80}\right)$ ranged from 9,6 to 11,5 days. The ABvPr11 strain presented the highest biocidal activity for the Schistocerca piceifrons peruviana L.A.
\end{abstract}

Key words: entomopatogen, biological control, locust, Schistocerca, Beauveria,

\section{Introducción}

Las langostas han ocasionado pérdidas económicas considerables en la agricultura a nivel mundial, especialmente en los países del continente africano y Asiático, (Showler, 1996). En Sudamérica, las langostas son conocidas en Colombia, Argentina, Paraguay, Uruguay y Bolivia (Beingolea, 1995; Chris, 1992). En el Perú han sido reportadas dos especies plagas de importancia para la agricultura: Schistocerca piceifrons peruviana plaga endémica en los departamentos de Ayacucho, Huancavelica, Apurímac y Cusco; y $S$. interrita registrada para Lambayeque y Cajamarca (SENASA, 2003). Schistocerca piceifrons peruviana, la "langosta migratoria del Perú y Ecuador" ha sido reportada desde 1901, en la zona andina del sur del Perú, donde vive de manera permanente y cuando tiene las condiciones óptimas para su crecimiento y desarrollo, causa severos daños. El control y eliminación de estos voraces insectos incluyen métodos mecánicos, químicos y físicos; los cuales generalmente son contaminantes y afectan a otros organismos. Una alternativa a estos métodos es el control biológico, que se basa en la manipulación de enemigos naturales, como los hongos entomopatógenos de insectos, entre los géneros más conocidos están Paecilomyces, Hirsutela, Verticillium, Beauveria y Metarhizium (France et el., 1999). Los dos últimos son considerados como agentes potenciales para el desarrollo de un micoinsecticida (Alves, 1986).
El objetivo del trabajo fue aislar, caracterizar y evaluar el efecto entomocida de algunas cepas nativas de Beauveria sp.

\section{Materiales y métodos}

\section{Aislamiento e Identificación de los hongos}

Individuos adultas y ninfas de diferentes estadios de Schistocerca piceifrons peruviana fueron colectados en su zona de reproducción, Provincia de Huamanga del Departamento de Ayacucho, a $2761 \mathrm{~m}$ de altitud, en junio y diciembre 2003. Los ejemplares fueron transportados al laboratorio donde se aislaron las cepas puras de hongos, que se conservaron en Agar Papa Dextrosa (APD) (Pariona, 2006).

La identificación del hongo hasta género se realizó de acuerdo a las características morfológicas mediante la utilización de la clave taxonómica propuesta por Egorova (1980), Barnet y Hunter (1998).

\section{Multiplicación del hongo}

Reactivación de las cepas. Las cepas identificadas como Beauveria, fueron reactivadas en placas Petri con medio Agar Saboruraud Glucosa (ASG), sellándose con parafilm y mantenidas a $23{ }^{\circ} \mathrm{C}$ y $88 \%$ de humedad por 10 días. 


\section{Preparación del sustrato final e inoculación.}

Se colocó $100 \mathrm{~g}$ de arroz en una bolsa de polipropileno de $0,5 \mathrm{k}$ con $20 \mathrm{~mL}$ de agua destilada, se autoclavo a 15 libras de presión y $120^{\circ} \mathrm{C}$ por 30 minutos, luego se inoculó en las bolsas un tercio del medio ASG con el hongo esporulado en placa petri, con 3 repeticiones para cada cepa; Se incubaron a temperatura ambiente $\left(20^{\circ} \mathrm{C}\right)$ y $88 \%$ de humedad por un periodo de 21 días, tiempo donde se obtuvo la mayor esporulación.

\section{Caracterización fisiológica.}

Se realizó mediante la evaluación del porcentaje de germinación y la producción de conidias.

Recuento de conidias: El recuento de conidias se realizó en la etapa de evaluación de la capacidad entomopatógena; para ello se preparo una solución conidial, en un tubo de prueba: se colocó $1 \mathrm{~g}$ del sustrato con las esporas, más $9 \mathrm{~mL}$ de agua destilada, más una gota del adherente agral al 0,5\%; se agitó vigorosamente y posteriormente se realizaron diluciones sucesivas hasta la dilución $10^{-3}$. Luego con una pipeta Pasteur, se tomó de la menor dilución una alícuota de la suspensión homogénea de conidias, se colocó en la ranura lateral de una cámara de Neubauer y se realizaron los conteos de conidias en 5 campos de la cámara para obtener el promedio de esporas de cada cepa en estudio.

Porcentaje de germinación de conidias: Para la cuantificación del porcentaje de germinación, se utilizaron placas Petri conteniendo papel filtro, portaobjeto estéril. Sobre la lámina se adicionó de 4 a 5 cuadriculas del medio de cultivo ASG, al cual se le añadió una gota de la dilución de esporas $\left(10^{8}\right.$ conidias $\left./ \mathrm{mL}\right)$, seguidamente se cubrió con una llaminilla; se incubó por 18 horas; finalmente se agregó una gota de azul de lactofenol para detener la germinación y dar contraste a las conidias. El conteo se realizó en 5 campos.

Evaluación de la capacidad biocida de las cepas nativas de Beauveria sp. sobre $S$. piceifrons peruviana.

Identificada la cepa como Beauveria sp. se evaluó la patogenecidad para seleccionar a la más eficiente. El ensayo se llevó a cabo en un ambiente abierto acondicionado con jaulas de $100 \times 50$ $\mathrm{cm}$, a temperatura y humedad ambientales, en el Laboratorio de Entomopatógenos del Servicio Nacional de Sanidad Agraria (SENASA-Ayacucho).

Cada experimento tuvo un diseño estadístico completamente al azar, con insectos adultos de $S$. piceifrons peruviana por cada cepa de hongo, la cual comprendió un tratamiento. Cada tratamiento fue evaluado con 50 individuos, en estado adulto, con 3 repeticiones cada una. Este bioensayo tuvo como finalidad seleccionar la cepa que ocasiona el mayor porcentaje de mortalidad. Para la prueba de patogenecidad de Beauveria sp. se prepararon cuatro soluciones de $10^{8}$ conidias $/ \mathrm{mL}$, a partir de la solución madre respectiva de las cuatro diferentes cepas del hongo a evaluar.

Se evaluaron cinco tratamientos:

\begin{tabular}{|c|c|c|c|c|c|}
\hline 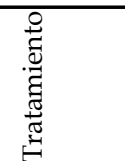 & 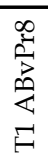 & 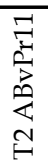 & 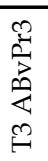 & 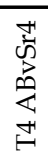 & 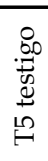 \\
\hline Adultos & 50 & 50 & 50 & 50 & 50 \\
\hline
\end{tabular}

La infección del insecto se hizo por aspersión, con un spray sobre los insectos, para el testigo se hizo el mismo tratamiento, pero la aspersión se hizo con solución acuosa estéril.

Las evaluaciones fueron diarias; una vez muerto el insecto se lavó por inmersión en hipoclorito de sodio al 5\%, por un minuto, se enjuagó en agua destilada estéril 3 veces y luego se colocó en cámara húmeda para facilitar el crecimiento de micelio. Seguidamente, se verificó si se trataba de la misma cepa nativa aislada del insecto, para ello se hizo un examen directo con azul de lactofenol observándose las estructuras de fructificación de los hongos al microscopio.

El diseño fue completamente al azar, se transformaron los datos obtenidos mediante la expresión $\operatorname{arcsen} \sqrt{\mathrm{m}}$, donde $\mathrm{m}$ es el valor de la variable porcentaje de individuos muertos. Los promedios se compararon mediante la prueba de Duncan $(\mathrm{P}<0,05)$. Los datos de mortalidad total acumulada de cada concentración fueron corregidos mediante la fórmula de Abbot.

\section{Tiempo de letalidad al 50 y $80 \%\left(\mathrm{TL}_{50}\right.$ y $\left.\mathrm{TL}_{80}\right)$ de la po- blación expuesta.}

Con esta variable se determino el tiempo que demora el hongo en matar al 50\% y al $80 \%$ de langostas en cada unidad experimental por medio de una tabla de acumulado diario durante 12 días; con estos datos se realizó el respectivo análisis de varianza (ANOVA), los promedios fueron comparados mediante el análisis de Duncan $(\mathrm{P}<0,05)$.

\section{Tasa de infección.}

Se seleccionaron las langostas muertas al final del experimento, se separaron en cámara húmeda para realizar un conteo de langostas infectadas por el hongo, para hallar el porcentaje de infección dentro de cada unidad experimental y tratamiento.

\section{Resultados y discusión}

\section{Aislamiento e Identificación de Cepas de Beauveria sp.}

Se aislaron 10 cepas de hongos, de las cuales 4 corresponden a Beauveria sp: ABvPr11, ABvPr8, ABvPr3 y ABvSr4. Los 4 aislamientos se obtuvieron a partir de langostas nativas de Ayacucho, es decir, que el hongo esta presente en ella en su forma natural. Así también, lo señalan Zimmerman (1992), Glare (1992), Castineiras et al. (1996), Alcázar et al. (2003).

\section{Caracterización morfológica.}

Las cepas $\mathrm{ABvPr} 11, \mathrm{ABvPr} 8, \mathrm{ABvPr} 3$ y $\mathrm{ABvSr} 4$ presentaron: colonias con micelios blancos de aspecto algodonoso, superficie semielevada, crecimiento moderado, estas características son variables, por lo que no son consideradas como únicos criterios para determinar la especie (Vargas, 2003), al caracterizar tres cepas de Beauveria brongniartii.

Todas las cepas formaron sinemas (estructuras alargadas ramificadas) reportadas por Vargas (2003) para Beauveria bassiana. Según Samson et al. (1988), Rodríguez y Del Pozo (2003); estas estructuras son eficientes para la dispersión de conidias, lo cual indica que estas cepas serian buenos controladores en el campo.

Las 4 cepas produjeron pigmentación amarilla difundiéndose en el medio, que evidencia la presencia de Bauvericina, toxina secretada durante el crecimiento, como también lo mencionan Gómez (1998) y Vargas (2003) para B. bassiana y B. brongniartti. 
Tabla 1. Tasa de mortalidad total causadas por las cepas de Beauveria sp.

\begin{tabular}{|c|c|c|c|c|c|c|c|c|}
\hline \multirow[t]{2}{*}{ Tratamiento } & \multicolumn{2}{|c|}{ Día 8} & \multicolumn{2}{|c|}{ Día 10} & \multicolumn{2}{|c|}{ Día 11} & \multicolumn{2}{|c|}{ Día 12} \\
\hline & $\%$ & arcsen & $\%$ & arcsen & $\%$ & arcsen & $\%$ & arcsen \\
\hline ABvPr11 & 41,00 & $0,69 \mathrm{BC}$ & 90,00 & $1,26 \mathrm{C}$ & 96.0 & $1,38 \mathrm{C}$ & 100,00 & $1,57 \mathrm{~B}$ \\
\hline ABvSr4 & 32,00 & $0,60 \mathrm{~B}$ & 74,00 & $1,03 \mathrm{~B}$ & 82,00 & $1,13 \mathrm{~B}$ & 91,00 & $1,27 \mathrm{~B}$ \\
\hline ABvPr8 & 44,00 & $0,72 \mathrm{C}$ & 72,00 & $1,02 \mathrm{~B}$ & 82,00 & $1,13 \mathrm{~B}$ & 89,00 & $1,25 \mathrm{C}$ \\
\hline ABvPr3 & 36,00 & $0,65 \mathrm{C}$ & 64,00 & $0,93 \mathrm{~B}$ & 76,00 & $1,06 \mathrm{~B}$ & 83,00 & $1,15 \mathrm{C}$ \\
\hline Testigo & 6,00 & $0,25 \mathrm{~A}$ & 14,00 & $0,36 \mathrm{~A}$ & 16,00 & $0,39 \mathrm{~A}$ & 18,00 & $0,39 \mathrm{~A}$ \\
\hline
\end{tabular}

Los valores de la misma letra no son significativos según la Prueba de Duncan $(P<0,05)$

Las características microscópicas observadas coinciden con las del género Beauveria sp.: conidióforos sencillos, agrupados irregularmente o en grupos verticilados, en algunas especies hinchados en la base y adelgazándose hacia la porción que sostiene la conidia, la cual se presenta en forma de zig-zag, las conidias son hialinas, redondeadas a ovoides y unicelulares coincidiendo con las descripciones de Bustillos (2001), Rodríguez y Del Pozo (2003). En nuestras cepas resaltan las características de B. bassiana que posee conidias globosas a subglobosas, las estructuras conidióforas forman densos grupos, como lo describe Samson et al. (1988). Por lo tanto, los aislados obtenidos podrían identificarse como B. bassiana. Sin embargo, aún se necesitan realizar más estudios fisiológicos y moleculares para asegurar su identidad.

\section{Caracterización fisiológica del hongo.}

Las cuatro cepas presentaron una alta producción de conidias después de 21 días de sembrado. Según el análisis de varianza (ANOVA) se determinó diferencias altamente significativas las cuales presentan valores mayores de $10^{8}$ conidias $/ \mathrm{mL}$. La cepa ABvSr4 presentó el valor mas alto con $10,9 \times 10^{8}$ conidias $/ \mathrm{mL}$, seguido de la cepa $A B v \operatorname{Pr} 8$ con $9,5 \times 10^{8}$ conidias $/ \mathrm{mL}$ y la cepa ABvPr 11 con $4,9 \times 10^{8}$ conidias $/ \mathrm{mL}$. El valor mas bajo lo presentó la cepa $A B v P r 3$ con $1,2 \times 10^{8}$ conidias $/ \mathrm{mL}$.

El porcentaje de germinación para las cuatro cepas de Beauveria sp. fueron del 95,4\%, 94,1\%, 94,7\%, 92,5\%, para las cepas ABVPr11, ABvPR3, ABvSr4 y ABvPr8 respectivamente. Según el Análisis de Varianza (ANOVA) no son significativamente diferentes $(\mathrm{P}<0,05)$.

La caracterización fisiológica del poder entomopatógeno, que se realizo a las 4 cepas en estudio fueron similares en sus porcentajes de germinación, no permitiendo establecer diferencias entre ellas, y en la producción de conidias mostró diferencias altamente significativas, resultados similares a los obtenidos por Vargas (2003), Bautista (1999) a las 24 y 21 horas respectivamente. Asimismo estos porcentajes obtenidos en el tiempo indicado coinciden con los requisitos descritos para el control de calidad de hongos entomopatógenos señalado por Gómez (1998).

Tabla 2. Tasa de mortalidad corregida según Abbot al día 10 y 12.

\begin{tabular}{ccc}
\hline Tratamiento & Mortalidad día12 & Mortalidad día11 \\
\hline ABvPr11 & 89,1 & 100 \\
ABvSr4 & 69,8 & 89,3 \\
ABvPr8 & 68,2 & 86,3 \\
ABvPr3 & 58,9 & 79,5 \\
\hline
\end{tabular}

\section{Prueba de patogenecidad.}

Todas las cepas produjeron síntomas iniciales como manchas oscuras en el tegumento y pérdida de movimiento que indican que las langostas están infectadas con el hongo. Los síntomas de la invasión del hongo en el hemocele, produce parálisis al insecto; esto explicaría la inmovilidad y rigidez observada en las langostas coincidiendo con las descripciones de Vargas (2003), Lecuona et al. (1996) quienes además, mencionaron que durante la germinación del hongo se produce una degradación enzimática de la cutícula, causada por la secreción de endoproteasas, quitinasas y lipasas, entre otras enzimas; la presencia de manchas necróticas evidencia sitios de penetración del hongo, como una respuesta a la acción lítica de las enzimas liberadas durante la penetración tal como lo menciona Bautista (1999) al evaluar la patogenicidad de $B$. bassiana sobre $S$. piceifrons peruviana, produciendo muerte a partir del segundo día.

El análisis de varianza (ANOVA) nos demuestra que existen diferencias significativas entre las cepas (ver Tabla 1).

Todas las cepas fueron altamente patogénicas. Sin embargo, hasta el octavo día no se observaron diferencias significativas de mortalidad entre las cepas. Al décimo día la cepa ABvPr11 con $90 \%$ de mortalidad, fue significativamente diferente a las cepas $\mathrm{ABvSr} 4$, ABvPr8 y ABvPr3, con 74\%, 72\% y 64,6\% de mortalidad y al testigo con un $14 \%$. El mismo comportamiento ocurre al onceavo día, aumentando los porcentajes de mortalidad en todos los tratamientos pero al doceavo día la cepa ABvPr11 logró matar al $100 \%$ de langostas, siendo significativamente diferente a las demás cepas $\mathrm{ABvSr} 4, \mathrm{ABvPr} 8$ y $\mathrm{ABvPr} 3$ las que alcanzaron el $91 \%, 89 \%$ y $83 \%$ respectivamente y el testigo con un porcentaje de $18 \%$.

Según la fórmula de Abbot se observa también que hay diferencias significativas en los últimos días (Tabla 2). Todas las cepas fueron altamente patogénicas llegando a matar al 100\% de las langostas, la diferencia varia en el tiempo. Para la cepa ABvPr11 fue al doceavo día, seguido de las cepas ABvPr8 y ABvPSr4 al catorceavo día y finalmente la cepa $\mathrm{ABvPr} 3$ al dieciseisavo día (Fig. 1) el análisis de varianza para esta variable de mortalidad total muestra diferencias significativas.

Al noveno día, estadísticamente no hay diferencias significativas de mortalidad en las cepas, esto se debe posiblemente a que los insectos mas débiles fueron muriendo primero y en los insectos normales la mortalidad se daría al décimo día. La cepa ABvPr 11 obtuvo una mortalidad de $90 \%$ con $10^{8}$ conidias $/ \mathrm{mL}$, mientras tanto, la cepa $\mathrm{ABvPr} 3$ obtuvo un porcentaje menor correspondiente al 64\%. La prueba de Duncan muestra dos grupos 


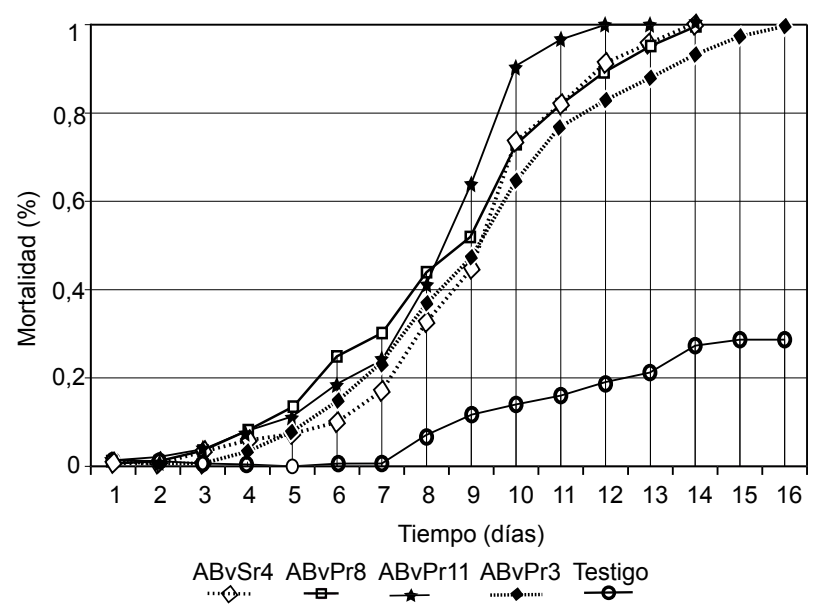

Figura 1. Prueba de patogenecidad de las cepas de Beauveria sp. sobre Schistocerca piceifrons.

significativamente diferentes coincidiendo con lo afirmado por Alves (1986), quien seńala que la patogenicidad es una característica genética de un microorganismo entomopatógeno, que lo lleva a penetrar en un insecto y causar la enfermedad, en tanto que la virulencia es el grado de patogenicidad de los aislamientos hacia un hospedero específico.

El presente trabajo fue realizado en jaulas en condiciones de temperatura y humedad ambientales. Las cepas produjeron la muerte del 76 al 96\% de población de langostas al onceavo día con concentraciones de $10^{8}$ conidias $/ \mathrm{mL}$, resultados similares obtuvieron por Foster et al. (1996) al evaluar B. bassiana sobre Melanoplus sanguinipus en jaulas de campo a temperatura y humedad ambientales durante 3 años, a concentraciones de $1 \times 10^{13}$ conidias $/ \mathrm{mL}$

La mortalidad de nuestras cepas ABvPr11 (100\%), ABvSr4 (91\%), ABvPr8 (89\%) y ABvPr3 (83\%) al doceavo día muestran resultados similares a los obtenidos por Gallegos et al. (2003) quienes evaluaron cepas nativas de B. bassiana en adultos de $\mathrm{Am}$ phidees sp. donde las cepas SAA (99\%) y HCA (97\%) produjeron mortalidades al treceavo día de la aplicación.

De la misma manera, nuestras cepas ABvPr11, ABvSr4, $\mathrm{ABvPr} 8$ y $\mathrm{ABvPr} 3$ evaluadas a condiciones ambientales, con alimento en floreros, con la concentración $10^{8}$ conidias $/ \mathrm{mL}$ y aplicaciones directas e indirectas causaron patogenecidad especifica de un $100 \%$ a los $12,14,14$ y 16 días respectivamente, coincidiendo con los resultados de Torres et al. (1997) en ensayos de $B$. bassiana, B. brongniartii y Metarh izium anisopliae en el control del "gusano blanco de la papa" (Premnotrypes vorax), que obtuvo

Tabla 3, Tiempo letal al 50 y $80 \%$ de la población de la cepas de Beauveria sp, $\left(\mathrm{TL}_{50}\right.$ y $\left.\mathrm{TL}_{80}\right)$

\begin{tabular}{ccc}
\hline Tratamiento & \multicolumn{2}{c}{ Tiempo letal } \\
& al 50\% & al 80\% \\
\hline ABvPr11 & $8,34 \mathrm{~A}$ & $9,6 \mathrm{~A}$ \\
ABvSr4 & $9,14 \mathrm{~B}$ & $10,8 \mathrm{~B}$ \\
ABvPr8 & $8,74 \mathrm{~A}$ & $10,8 \mathrm{~B}$ \\
ABvPr3 & $9,13 \mathrm{~B}$ & $11,5 \mathrm{~B}$ \\
\hline
\end{tabular}

Valores con la misma letra no presentan diferencias significativas según el análisis de Duncan $(P<0,05)$, ANOVA $(P<0,05)$ hasta un $100 \%$ de mortalidad después de 21 días de aplicados los tratamientos, a concentraciones de $1 \times 10^{9}$ conidias $/ \mathrm{mL}$.

Las cepas en estudio provienen del insecto a controlar, y por consiguiente el mejor potencial patogénico que demuestran en las pruebas, podría deberse a que estas cepas han sufrido procesos de selección natural y coevolución con el insecto, factores que pueden generar en la cepas una mayor especificidad en la infección del insecto blanco (Torres et al. 1997; France et al., 1999).

Tiempo de letalidad al 50 y $80 \%\left(\mathrm{TL}_{50}\right.$ y $\left.\mathrm{TL}_{80}\right)$ de la población expuesta.

Según el análisis de varianza (ANOVA) el tiempo necesario para eliminar al $50 \%$ de la población fue altamente significativa. Siendo el $\mathrm{TL}_{50}$ de 8,34; 8,74; 9,13 y 9,14 días para $A B v \operatorname{Pr} 11$, ABvPr8, ABvPr3 y ABvSr4 respectivamente. La cepa ABvPr11 es significativamente diferente a las demás cepas, mostró ser más efectivo al actuar en menor tiempo (8,34 días) en promedio, mientras que las otras cepas son iguales estadísticamente. El tiempo necesario para eliminar el $80 \%$ de la población también fue altamente significativo. Se observa que los registros para las cepas son de 9,6 para $A B v \operatorname{Pr} 11 ; 10,8$ para $A B v S r 4 ; 10,81$ para ABvPr8 y 11,5 para ABvPr3. En el análisis de Duncan la cepa ABvPr11 muestra una diferencia altamente significativa respecto a las demás cepas, eliminando en 9,6 días el 80\% (Tabla 3).

El TL ${ }_{50}$ hallado para las cuatro cepas de Beauveria sp. en S. piceifrons peruviana, tuvo valores entre 8,3 y 9,16 días, no habiendo diferencia significativa con los obtenidos por Luna y Lecuona (2001) quienes obtuvieron $\mathrm{TL}_{50}$ entre 7,2 y 9,2 días para el control de "tucuras", con Beauveria y Metarhizium, lo que indica que estas tienen buen potencial patogénico.

\section{Tasa de infección.}

La aparición del micelio ocurrió a las 48 horas de muerte del insecto al ser colocado en cámara húmeda. Las cepas esporularon en los cadáveres de la langosta, adquiriendo un aspecto polvoriento, observándose además la formación de sinemas. Se observó que la cepa con más alta tasa de infección fue ABvPr 11 con $94 \%$ y la que registro menor tasa fue la cepa $\mathrm{ABvPr} 3$ con $84 \%$ y para las otras 2 fue de $88 \%$ (ABvSr4) y 90\% (ABvPr8) (Fig. 2).

En la tasa de infección para nuestras cepas, las características observadas como la aparición del micelio se dio después de las 48 horas de muerte del insecto al ser colocado en cámara húmeda; lo que indicaría que la muerte fue causada por el hongo (Robert y Humber, 1984; Kershaw et al. 1999). Las cepas esporularon en los cadáveres de la langosta, adquiriendo un aspecto polvoriento, observándose

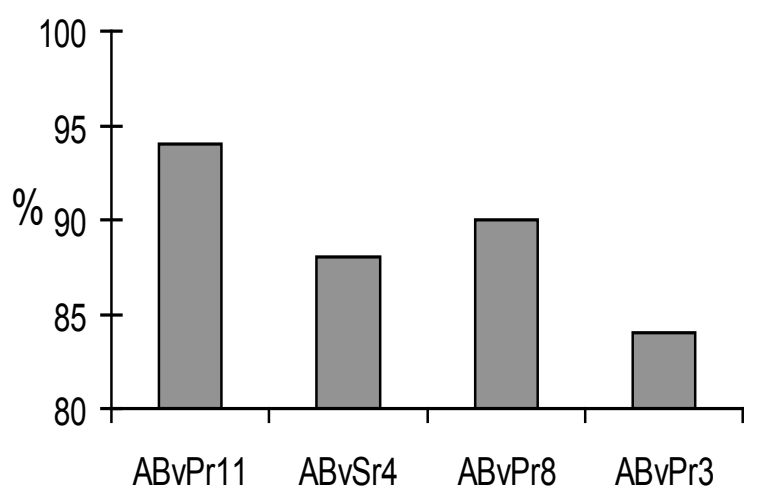

Figura 2. Porcentaje de tasa de infección de las cuatro cepas de Beauveria sp. 
además la formación de sinemas coincidiendo con las descripciones de Tanada y Kaya (1993) para el género Beauveria sp.

Se registraron porcentajes de micosis alta en la langosta por la cepa $A B v \operatorname{Pr} 11$ con $94 \%$ y menor porcentaje de micosis fue en la cepa $\mathrm{ABvPr} 3$ con $84 \%$ y para las otras 2 cepas fueron de $88 \%$ (ABvSr4) y $90 \%$ (ABvPr8).

\section{Literatura citada}

Alcázar, S.; V. Raman, E. Torres \& E. Yabar. 2003. Beauveria sp: Hongo amigo del agricultor. Boletín de la Papa. Centro Internacional de la Papa. Lima Perú. 5(1): 44-46.

Alves, S. 1986. Fungos entomopatogénicos. Control microbiano de insectos. Editorial Manole LTDA. Sao Paulo - Brasil. p: 278-288.

Barnett, H. L. \& Hunter, B. 1998. Illustrate general of imperfect fungi. 4ta Edición. The American Phytopathological Society. St Paul, Minnesota (USA). 217pp.

Bautista, N. 1999. Evaluación de la patogenicidad de las cepas Beauveria bassiana y Metarhizium anisopliae sobre Schistocerca piceifroms peruviana. En condiciones de laboratorio Ayacucho. Tesis. Universidad San Cristóbal de Huamanga Ayacucho - Perú. 44pp.

Beingolea, O. 1995. Langostas y su control. Ed. RAAA/SENASA, Lima. 143pp.

Bustillos, A. 2001. Hongos en insectos y posibilidades de uso en el control biológico de plagas en Colombia. En: Seminario sobre uso de entomopatógenos en Colombia. Sociedad Colombiana de Entomología. Bogotá. p: 30-53.

Castineiras, A.; J. E. Peña, R. Duncan \& L. Osborne. 1996. Potential of Beauveria bassiana and Paecilomyces fumosoroseus (Deuteromycotina: Hyphomycetes) as biological control agents of Thrips palmi (Thysanoptera: Thripidae). Florida Entomologist. 79(3): 458-461.

Chris P. 1992. Los Bioplaguicidas contra las langostas. Mundo científico 34(13): 369-370.

Egorova, L. N. 1980. Hongos del suelo del Lejano este. Hyphomycetes. Instituto de Biología del Suelo. Centro Científico del Lejano Este. Academia de Ciencias. URSS. Editorial "Ciencia", División de Leningrado. p: 49 -76 (en ruso)

Foster, R. N.; C. Reuter, J. Britton, \& C. Bradley. 1996. Lab studies and field trials with the fungus Beauveria bassiana against grasshoppers. Mycotech Corporation. U.S.A. p: 5-8.

France, A., M. Gerding, A. Sandoval, S. Espinosa y E. Vivanco. 1999. Patología de Insectos. Serie Quilamapu N. ${ }^{\circ} 122$. Ed. INIA Quilamapu, Chillán, Chile. $119 \mathrm{p}$

Gallegos, G.; R. Olayo, E. Guerrero, M. Sánchez, M. Cepeda, Y. Grant \& H. Quiroz. 2003. Evaluación de cepas nativas de Beauveria bassianal (vuill.) sobre Amphidees sp. de Arteaga, Coahuila. En Resultados de Proyectos de Investigacion 2003, Direccion de Investigaciones, Universidad Autonoma Agraria Antonio Narro. Mexico. Pp: 360-367, En Linea http://www.uaaan.mx/DirInv/Rdos2003/parasiagric/evcepas.pdf (acceso 13/02/07)

Glare R. T. 1992. Fungal pathogens of scarabs. In: Use of pathogens in scarabs pest management. Jackson, T. A. (ed.). R.T.London: Atheneaum press. p: 63-79.
Gómez, H. 1998. Importancia de los hongos entomopatógenos. En: Nuevos Aportes del Control Biológico en la Agricultura Sostenible. Editores: A. Lizárraga T., U. Barreto C. y J. Hollans. Red de Acción en Alternativas Al uso de Agroquímicos. p: 97-112.

Kershaw, J.; E. R. Moorhouse, R. Bateman, S. E. Reynolds \& A. K. Charnley. 1999. The role of destruxin in the pathogenicity of Metharhizium anisoplae for three species of insects. Journal of Invertebrate Pathology. 74: 213-223.

Lecuona, R. E.; B.Papierok, \& Riba. 1996. Hongos entomopatógenos. En: Microorganismos patógenos empleados en el control microbiano de insectos plaga. R.E. Lecuona (ed). Buenos Aires, Argentina. p: 36-60.

Luna, R. J. A. \& R. E. Lecuona. 2001. Selección de hongos entomopatógenos nativos para el control de la tucura Rhammatocerus pictus (Bruner) (Orthoptera: Acrididae) INTA. Argentina, RIA, 31(1):67-84.

Pariona M., N. 2006. Evaluación de la capacidad entomocida de Beauveria sp. SOBRE Schistocerca piceifrons peruviana (Lynch Arribalzaga, 1903) nativos del departamento de ayacucho. Tesis titulo de Biólogo. Facultad de Ciencias Biológicas, Universidad Nacional Mayor de San Marcos. Lima-Perú. 51pp.

Roberts, D. \& R. Humber. 1984. Entomopathogenic fungi. In Infection proceses of fungy. A Bellagio conference, Roberts, D; Aisy, J. (Eds). March 21- 25, 1983. The Rockefeller Foundation. New York. p: 1-12.

Rodríguez, A. \& E. Del Pozo. 2003. Aislamiento de hongos entomopatógenos y evaluaron su virulencia sobre Trialeurodes vaporariorum (west). Agrociencia. 7(2): 71-78.

Samson, R.; H. Evans \& J.Latge. 1988. Atlas of Entomopathogenic Fungi. Springer-Verlag, Berlin. 300 pp.

Servicio Nacional de Sanidad Agraria (SENASA) 2003. Manejo integrado de la plaga de la langosta. Programa Nacional de Control Biológico. http://www.senasa.gob.pe/sanidad_vegetal/programas_fitosanitarios/mip_langosta/index. htm (acceso 13/02/07)

Showler, A. T. 1996. La Langosta del Desierto en África y Asia Occidental: Complejidades de Guerra, Política, Terreno Peligroso, y Desarrollo. En: E. B. Radcliffe y W. D. Hutchison [eds.], Radcliffe: Texto Mundial de MIP, URL: $<$ http://ipmworld.umn.edu $>$, Universidad de Minnesota, St. Paul, MN. (acceso 13/02/07)

Tanada, Y. \& H. Kaya. 1993. Insect Pathology. Academic Press. San Diego, California. (USA). 666 pp.

Torres, R. \& A. López. 1997. Estudios básicos para el control microbiológico del gusano blanco de la papa (Premnotrypes vorax) con Beauveria sp. y Metarhizium sp. Tesis. Facultad de Ciencias, Pontificie Universidad Javeriana. Santa Fe - Bogotá. 33: 76pp.

Vargas, M. 2003. Caracterización de tres cepas de Beauveria brongniartii (saccardo) petch y su virulencia en Phthorimea operculella (Séller) y Symmetrischema tangolias (Gyen). Tesis. Facultad de Ciencias Biológicas, Universidad Nacional Mayor de San Marcos. Lima-Perú. 78pp.

Zimmerman, G. 1992. Metarhizium anisopliae an entomopathogenic fungus. Pflanzenschutz-Nachrichten Bayer. 45 (1):113 $-128$ 


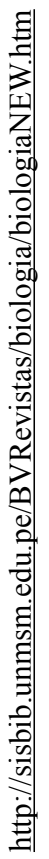

